\title{
Influência na qualidade de vida dos estudantes de Medicina relacionadas a má
}

\section{alimentação e sono}

\author{
Influence on the quality of life of Medicine students related to bad food and sleep \\ Influencia en la calidad de vida de estudiantes de Medicina relacionada con la mala comida y el \\ sueño
}

\section{Resumo}

A má qualidade de vida de estudantes da área da saúde está intimamente relacionada a algumas variáveis, tais como transtornos de alimentação e do sono. Estudantes da área da saúde recebem informações sobre nutrição adequada, no entanto, isso não implica que esses conhecimentos sejam seguidos por comportamentos alimentares apropriados. Em relação ao sono que é um elemento fundamental para a saúde do ser humano, visto que está envolvido em múltiplos mecanismos fisiológicos sistêmicos, sabe-se que a necessidade fisiológica do sono varia muito conforme a idade. Normalmente, adultos jovens necessitam, em média, de sete a nove horas de sono por noite. Dessa forma, o presente estudo tem por objetivo elucidar os principais fatores relacionados a má qualidade da alimentação e do sono nos estudantes da área da saúde e as consequências desses hábitos no organismo e no rendimento acadêmico. Foi realizada uma revisão integrativa de estudos da literatura obtidos por buscas nas bases de dados: PubMed, SciELO e BVS, utilizando os descritores: comportamento alimentar, estudantes de medicina, distúrbios do sono. Foram incluídos estudos publicados entre 2015 e 2020, em língua portuguesa, inglesa e espanhola. A partir desses dados foi feita uma compilação das informações mais relevantes para a construção do presente trabalho. Foi verificado que estudantes do curso de medicina apresentam hábitos alimentares pouco saudáveis, sendo caracterizados por baixo consumo de vegetais, frutas e água, e uma maior ingesta de carne vermelha, comidas industrializadas e doces. A literatura mostra uma estreita correlação entra a imagética corporal e o comportamento alimentar. A má qualidade no sono está correlacionada a aprendizagem superficial dos estudantes universitários da área da saúde. Há também uma estreita ligação entre a breve duração de sono e maiores chances de desenvolver hiperlipidemias, acidentes automobilísticos e 
doenças arteriais coronarianas. Foram apresentados os diversos fatores de risco para doenças crônicas não transmissíveis em estudantes da área da saúde, dentre as quais destacam-se neoplasias, distúrbios metabólicos, doenças dos sistemas nervoso, respiratório, cardiovascular e osteoarticular, principalmente. Assim, os resultados evidenciam as diferenças entre o estilo de vida e o recomendado, sobretudo em relação ao sono e a ingesta de alimentos. Esta pesquisa contribui com a obtenção de mais informações sobre os fatores que influenciam no processo de adoecimento em estudantes universitários, sobretudo acadêmicos da área da saúde.

Palavras-chave: Comportamento alimentar; Distúrbios do sono; Cefaleia; Obesidade.

\begin{abstract}
The poor quality of life of health students is closely related to some variables, such as eating and sleeping disorders. Health students receive information about adequate nutrition; however, this does not imply that this knowledge is followed by appropriate eating behaviors. Regarding sleep, which is a fundamental element for human health, since it is involved in multiple systemic physiological mechanisms, it is known that the physiological need for sleep varies a lot according to age. Typically, young adults need, on average, seven to nine hours of sleep per night. Thus, the present study aims to elucidate the main factors related to poor quality of food and sleep-in health students and the consequences of these habits on the body and academic performance. An integrative review of studies of the literature was obtained by searching the databases: PubMed, SciELO and VHL, using the descriptors: eating behavior, medical students, sleep disorders. Studies published between 2015 and 2020 in Portuguese, English and Spanish were included. From these data, a compilation of the most relevant information for the construction of this work was made. It was found that medical students have unhealthy eating habits, being characterized by low consumption of vegetables, fruits and water, and a greater intake of red meat, processed foods, and sweets. The literature shows a close correlation between body imagery and eating behavior. Poor sleep quality is correlated with superficial learning by university students in the health field. There is also a close link between short sleep duration and increased chances of developing hyperlipidemia, car accidents and coronary artery disease. The various risk factors for chronic noncommunicable diseases in health students were presented, among which neoplasms, metabolic disorders, diseases of the nervous, respiratory, cardiovascular and osteoarticular systems stand out, mainly. Thus, the results show the differences between lifestyle and what is recommended, especially in relation to sleep and food intake. This research contributes to obtaining more information about the factors that influence the illness process in university students, especially academics in the health area.
\end{abstract}

Keywords: Eating behavior; Sleep disorders; Headache; Obesity.

\title{
Resumen
}

La mala calidad de vida de los estudiantes de salud está estrechamente relacionada con algunas variables, como los trastornos alimentarios y del sueño. Los estudiantes de salud reciben información sobre una nutrición adecuada, sin embargo, esto no implica que este conocimiento sea seguido por conductas alimentarias adecuadas. En cuanto al sueño, que es un elemento fundamental para la salud humana, ya que está involucrado en múltiples mecanismos fisiológicos sistémicos, se sabe que la necesidad fisiológica de sueño varía mucho según la edad. Por lo general, los adultos jóvenes necesitan, en promedio, de siete a nueve horas de sueño por noche. Así, el presente estudio tiene como objetivo dilucidar los principales factores relacionados con la mala calidad de la alimentación y el sueño en los estudiantes de salud y las consecuencias de estos hábitos en el organismo y el rendimiento académico. Se obtuvo una revisión integradora de estudios de la literatura mediante la búsqueda en las bases de datos: PubMed, SciELO y VHL, utilizando los descriptores: conducta alimentaria, estudiantes de medicina, trastornos del sueño. Se incluyeron estudios publicados entre 2015 y 2020 en portugués, inglés y español. A partir de estos datos se realizó una recopilación de la información más relevante para la construcción de esta obra. Se encontró que los estudiantes de medicina tienen hábitos alimentarios poco saludables, caracterizándose por un bajo consumo de verduras, frutas y agua, y una mayor ingesta de carnes rojas, alimentos procesados y dulces. La literatura muestra una estrecha correlación entre las imágenes corporales y la conducta alimentaria. La mala calidad del sueño se correlaciona con el aprendizaje superficial de los estudiantes universitarios en el campo de la salud. También existe un vínculo estrecho entre la corta duración del sueño y el aumento de las posibilidades de desarrollar hiperlipidemia, accidentes automovilísticos y enfermedad de las arterias coronarias. Se presentaron los diversos factores de riesgo de enfermedades crónicas no transmisibles en estudiantes de salud, entre los que destacan, principalmente, las neoplasias, trastornos metabólicos, enfermedades del sistema nervioso, respiratorio, cardiovascular y osteoarticular. Así, los resultados muestran las diferencias entre el estilo de vida y lo recomendado, especialmente en relación con el sueño y la ingesta alimentaria. Esta investigación contribuye a obtener más información sobre los factores que influyen en el proceso de la enfermedad en estudiantes universitarios, especialmente académicos del área de la salud.

Palabras clave: Comportamiento alimentario; Trastornos del sueño; Dolor de cabeza; Obesidad. 


\section{Introdução}

A organização mundial da saúde (OMS) define a qualidade de vida como a percepção de cada indivíduo quanto a sua posição na sociedade, em relação à cultura e sistemas de valores, incluindo expectativas e preocupações. Isso demonstra o quão amplo é o conceito e o quanto ele é influenciado pela saúde tanto física quanto mental. Diante disso, evidências sugerem que à má qualidade de vida de estudantes da área da saúde está intimamente relacionada a algumas variáveis, tais como, transtornos de alimentação e do sono (Qiu et al., 2019).

O período ou o semestre letivo em que o acadêmico se encontra dita as atitudes de saúde tomadas por ele, influenciando diretamente na qualidade de vida como um todo. Isso é um fator que molda completamente a maneira como o indivíduo se adequa as exigências impostas. Em contrapartida, ainda hoje os estudos não se atentaram em relacionar o período ou semestre do curso com as diferentes maneiras que afetam a vida do acadêmico (Jaremków et al., 2020).

Sabe-se que os estudantes da área da saúde possuem informações acerca da nutrição adequada, porém, isso não implica que esses conhecimentos sejam seguidos por comportamentos alimentares apropriados, embora esses acadêmicos, geralmente, demonstrem melhores hábitos em relação a estudantes de outras áreas (Ferreira et al., 2020). Além disso, estudantes de medicina, quando comparados com estudantes de mesma idade, mas de áreas diferentes, suportam maior estresse emocional às mudanças de hábitos de vida, podendo, muitas vezes, estar menos suscetíveis aos distúrbios alimentares e de sono (Bezerra et al., 2020).

A obesidade ocorre quando a ingestão de energia excede sua produção por um longo período. Esse desequilíbrio, comumente, é causado por ingestão excessiva de alimentos e/ou atividade física inadequada, mas outros fatores do estilo de vida, tais como má alimentação e higiene do sono, podem desencadear ou acelerar esse processo. Em relação ao sono, evidências recentes apontam que o sono e o aumento de peso são fatores inversamente proporcionais, uma vez que pessoas cujas o sono é mais curto que o normal, têm mais tempo para comer e por isso aumentar sua ingestão de calorias, geralmente com alto teor de gordura, sal e carboidratos (Kanikowska et al., 2017).

Nessa perspectiva, os distúrbios do sono, da dieta e exercícios inadequados estão relacionados ao excesso de peso. Esse panorama pode ser a base para doenças psicossomáticas, tais como ansiedade, distúrbios metabólicos, incluindo resistência à insulina e obesidade, e aumento do risco cardiovascular. Os problemas da obesidade e os riscos associados aos distúrbios metabólicos e cardiovasculares encontrados em adultos podem começar na infância, exceto em algumas situações, em que mudanças estressantes na vida pessoal, como o ingresso na vida acadêmica, podem gerar transtornos que irão resultar em comportamentos prejudiciais à saúde (Torres-Mallma et al., 2016; Ferreira et al., 2020).

Referente ao sono, esse é um elemento fundamental para a saúde do ser humano, visto que está envolvido em múltiplos mecanismos fisiológicos sistêmicos, como metabolismo, regulação do apetite, funções hormonais e imunológicas, além de regular os sistemas cardiovascular e neurológico, interferindo na boa memorização, sendo considerado um fator determinante da limpeza de resíduos cerebrais, o que contribui diretamente para o estado fisiológico como uma homeostasia essencial do organismo. Com isso, pessoas que dormem e acordam mais cedo têm melhor rendimento mental e cognitivo em relação a pessoas que atrasam o sono (Mirghani et al., 2019).

A necessidade fisiológica do sono varia muito conforme a idade, embora considere-se que, normalmente, os adultos jovens necessitam, em média, de sete a nove horas de sono por noite. Diante disso, a nova geração, denominada geração $\mathrm{Y}$ ou geração do milênio, passam várias horas do dia destinadas aos meios tecnológicos, seja para estudo ou lazer. Esse quadro influencia negativamente a saúde, ao afetar as horas de sono necessárias para um bom funcionamento orgânico, influenciando até mesmo nos relacionamentos sociais (Hernández et al., 2019).

O sono insuficiente é um dos principais fatores relacionados à cefaleia nos universitários, além do excesso de estudo, estresse, ansiedade e maus hábitos alimentares, inclusive o uso abusivo de cafeína ou demais substâncias psicoativas (Carneiro 
et al., 2019). Isso ocorre, sobretudo, em períodos de provas, uma vez que a grande quantidade de avaliações são fatores estressantes para os universitários, além da utilização de dispositivos emissores de luz na hora de dormir, como celular e computador, gerando alterações do ciclo sono-vigília (Santos et al., 2019). Desse modo, a má qualidade do sono pode causar déficits de atenção e falha no desenvolvimento acadêmico, além de ser um fator de risco para possíveis manifestações prodrômicas de diversas doenças (Abdulah \& Piro, 2018; Bezerra et al., 2020).

A cefaleia é considerada uma das principais queixas dos estudantes da área da saúde, uma vez que está relacionada à carga horária exaustiva e aos fatores estressores. Sabe-se que a percepção da dor é influenciada por processos fisiológicos, psicológicos e sociais. Assim, as emoções têm papel essencial nesse processo, uma vez que interferem na modulação dolorosa (Carneiro et al., 2019). Somando-se a isso, a má higiene do sono pode favorecer o surgimento de apneia obstrutiva do sono, insônia, narcolepsia, isto é, sonolência diurna em excesso, transtorno periódico de movimento de membros durante a noite, como a síndrome das pernas inquietas e irregularidade no ciclo circadiano biológico, interferindo nas atividades diurnas (Mirghani et al., 2015; Gonçalves; Haas, 2020).

Vale ressaltar que, conselhos para melhorar a dieta e a higiene do sono têm se tornado importante. Esse cenário de conscientização torna-se extremamente relevante quando se trata dos futuros profissionais da saúde, uma vez que são modelos a serem seguidos pelos seus pacientes (Kanikowska et al., 2017). Essa pesquisa limitará à qualidade de vida dos universitários da área da saúde, visto que são uma população vulnerável à aquisição desses fatores de risco e, ao mesmo tempo, privilegiados, pois têm a oportunidade de aprender sobre conteúdos relacionados a estilos de vida saudáveis em detrimento do restante da população (Choque Zurita et al., 2018).

Esta pesquisa contribui com a obtenção de mais informações sobre os fatores que influenciam no processo de adoecimento em estudantes universitários, sobretudo acadêmicos da área da saúde. Com isso, o objetivo do presente estudo é elucidar os principais fatores relacionados à má qualidade da alimentação e do sono nos estudantes da área da saúde e suas consequências no organismo e no rendimento acadêmico.

\section{Metodologia}

Trata-se de uma revisão integrativa de literatura, que permite a síntese de diversos estudos publicados, possibilitando conclusões a respeito de uma área específica (Pereira et al., 2018). A questão norteadora dessa pesquisa foi: "Qual é a relação dos maus hábitos alimentares e do sono em estudantes da área da saúde com as alterações no organismo e no rendimento acadêmico?". Para responder tal questionamento, foram selecionados 25 artigos, dos quais 18 em língua inglesa, 5 em língua espanhola e 2 em língua portuguesa. Os critérios de inclusão foram estudos realizados em seres humanos, publicados entre os anos de 2015 e 2020, que trouxessem dados referentes à alimentação e sono de estudantes universitários e artigos publicados e indexados nos seguintes bancos de dados: United States National Library of Medicine (PubMed), Biblioteca Virtual em Saúde (BVS) e Scientific Eletronic Library Online (SciELO). Além disso, os Descritores em Ciências da Saúde (DeCS) utilizados foram: "Eating behavior and medical students" e "Sleep disorder and medical students" e seus correspondentes na língua portuguesa. Os critérios de exclusão foram estudos que envolvessem estudantes que não fossem da área da saúde, produções que envolviam estudantes do ensino fundamental e médio, e trabalhos com amostras insuficientes e não publicados nos últimos cinco anos. O artigo foi lido na íntegra para determinar sua elegibilidade para posterior inclusão no estudo. Caso contrário, essas produções foram excluídas.

\section{Resultados e Discussão}

Um estudo demonstrou que, em estudantes do curso de medicina, os hábitos alimentares, geralmente, são pouco saudáveis, sendo caracterizados por baixo consumo de vegetais, frutas e água, e uma maior ingesta de carne vermelha, comidas 
industrializadas e doces. Essa irregularidade na alimentação, provavelmente, está associada ao maior risco de desenvolvimento de doenças crônicas não transmissíveis, como os diferentes tipos de neoplasias, diabetes melito, hipertensão arterial, doenças cardiovasculares, doenças respiratórias e doenças osteoarticulares (Choque Zurita et al., 2018). Outrossim, o curso de medicina é conhecido como um gerador de pressão e esgotamento nos estudantes, seja pela competitividade entre os próprios alunos, seja pela carga horária excessiva, o que pode gerar sentimento de insegurança, autodúvida e tristeza, constantemente. Todos esses fatores, estressores em conjunto, contribuem para desencadear, além das doenças já mencionadas, cefaleia, que é uma queixa comum nessa população universitária (Carneiro et al., 2019).

Uma pesquisa realizada com 384 acadêmicos, sendo 218 calouros e 166 estudantes do sexto ano, mostrou que, os calouros têm maior costume de se alimentar em casa, um percentual maior do que os alunos do sexto ano, embora essa diferença seja pequena (57,8\% a 50\%). Quanto ao consumo diário, a porcentagem de estudantes que consomem três ou mais refeições foi de 11,5\% entre os iniciantes e 18,7\% entre os do sexto ano. Entretanto, os veteranos consomem maior quantidade de fast food em relação aos alunos do primeiro ano, $18,7 \%$ e 13,3\% respectivamente, mesmo que seja claro para esses estudantes a recomendação mínima de três refeições por dia para manter os níveis glicêmicos necessários. Nesse mesmo pensamento, é ressaltado que os alunos do primeiro ano estariam mais suscetíveis a problemas alimentares, provavelmente porque eles precisam se adaptar de maneira abrupta a um novo estilo de vida adequando a sua dieta, a qual, geralmente, é constituída por fast foods e outros alimentos com substâncias calóricas e prejudiciais à saúde (Torres-Mallma et al., 2016).

De 100 pessoas entrevistadas em uma pesquisa, sendo 43\% estudantes de medicina, 69 mulheres e 31 homens, um total de 50,7\% no sexo feminino e 48,3\% no sexo masculino, substituem o almoço por fast food. Esse consumo de lanches, é muito comum em estudantes universitários, uma vez que a maioria dos alunos não tomam café da manhã de maneira adequada devido à falta de tempo e por esses alimentos serem de rápido e fácil acesso. Contudo, os estudantes de medicina entrevistados apresentaram maior conhecimento, em relação a outros indivíduos pesquisados, de que os fast foods causam doenças por fornecerem alto teor calórico e ingredientes nocivos à saúde, mas, mesmo assim, admitem o consumo alegando não terem outra opção (Tamayo et al., 2016).

Um estudo mostra ainda a relação da moradia com os hábitos alimentares das pessoas pesquisadas. Cerca de $36 \%$ desses indivíduos que moram com os familiares consomem refrigerante apenas uma vez na semana, $29 \%$ consomem duas vezes por semana, mas, os que moram na ausência dos pais consomem no mínimo duas vezes semanalmente. Dessa forma, a influência da família é fundamental para a formação e desenvolvimento de bons hábitos alimentares, já que, sem a supervisão dos pais, a tendência de se consumir alimentos menos saudáveis é visivelmente maior. Essa substituição alimentar, seja no café da manhã, almoço ou janta, foi observada de maneira clara nos estudantes, em ambos os sexos, tendo como fator causal, de acordo com a pesquisa em questão, o fator estressante de ser acadêmico e à baixa disponibilidade econômica por morar sozinho, optando por alimentos rápidos e baratos, mas pobres em nutrientes (Tamayo et al., 2016).

Em contrapartida, uma outra análise elaborada com 369 estudantes de ciências da saúde, sendo 319 mulheres e 50 homens, demonstrou que, de maneira generalizada, não há detecção de diferenças significativas quanto ao tipo de universidade, residência atual e condição de vida no que diz respeito ao comportamento alimentar. No entanto, quando analisados antes de ingressar na faculdade e comparados depois desse ingresso, os estudantes mostraram diferenças quanto ao apetite, atividades preferidas no tempo livre e tipos de refeições. Apesar disso, ainda que os estudos sejam controversos, cerca de 63\% da amostra estavam na categoria normal de Índice de Massa Corporal (IMC), enquanto 32,2\% estariam com sobrepeso e 4,2\% como baixo peso, o que, de certo modo, comprova que mesmo que exista uma alteração alimentar com a mudança para a vida universitária, essa não se faz de maneira tão grosseira a ponto de gerar alterações significativas no IMC, mas sim, são considerados hábitos alimentares não saudáveis, como, por exemplo, pular refeições (El-Kassas \& Ziade, 2016).

Uma avaliação feita quanto à atividade física, sobrepeso e obesidade com 251 estudantes de medicina, em que $60 \%$ 
eram do primeiro ao terceiro ano e $40 \%$ do quarto ao sétimo ano, apenas 7,2\% comprovaram nunca ter consumido fast food, o que demonstra a presença de maus hábitos alimentares na grande parcela pesquisada. Para tanto, essa concepção é motivada pela restrição de tempo para o preparo de refeições mais saudáveis devido às atividades educativas exigentes. Dentre os 251 estudantes avaliados, 169 eram mulheres e 82 eram homens. A prevalência de sobrepeso ou obesidade foi de $50 \%$ entre os estudantes do sexo masculino e de $32,7 \%$ entre o sexo feminino. Entretanto, cerca da metade dos estudantes analisados estava tentando perder peso, e a maioria tinha a crença de que tanto o controle dietético quanto o exercício eram importantes para a perda de peso, o que mostra que o conhecimento acerca da saúde adquirido por eles, os fazem conscientes de que podem estar com sobrepeso (Ahmed et al., 2019).

Conforme o estudo de Kowalska et al. (2017), acerca de substâncias nocivas comumente encontradas nos produtos alimentícios, a acrilamida, presente em bebidas como o café e alimentos ricos em carboidratos, como a batata frita e pão, está relacionada ao risco para a saúde humana por influência neurotóxica e carcinogênica. Essa pesquisa mostrou que, em relação à maioria da população, os estudantes universitários consomem menos esses alimentos com acrilamida, o que pode ser efeito de uma maior conscientização dos acadêmicos em relação ao restante da sociedade. Em contrapartida, o consumo do café, que também é fonte dessa substância em questão, é uma bebida muito consumida pelos estudantes como meio energético para a manutenção de maior quantidade de horas em estudo (Kowalska et al., 2017).

Uma outra análise feita com 710 estudantes, dentre eles, cerca de 464 mulheres e 246 homens, com média de 21 anos, demonstrou que desses, $11,1 \%$ eram baixo peso, 13,4\% estavam acima do peso e 1,8\% eram obesos. Além disso, a prevalência de distúrbios alimentarem foi de $32,8 \%$, sendo $37,6 \%$ nas mulheres e $23,7 \%$ nos homens. Contudo, o comportamento de controle de peso foi observado em 18,5\% dos estudantes, sendo que 6,5\% declararam estar em dieta, 7\% em jejum, 3\% em uso de supressores de apetite e 1,7\% usando a indução do vômito, além da presença de uso de laxantes e diuréticos. Isso mostra o quanto o conhecimento na área da saúde, ao mesmo tempo em que colabora para uma boa alimentação, pode ser prejudicial quando se usa de artifícios não benéficos ao organismo (Azzouzi et al., 2019).

Um trabalho referente aos aspectos da imagem corporal, elaborou uma proposta em que foram usados sete modelos anatômicos para ambos os sexos com o respectivo $\operatorname{IMC}(18,22,25,27,30,35$ e 40). Além disso, havia a seguinte pergunta: "quando você se olha nu no espelho, com qual imagem você se parece?". Posteriormente, a resposta da pessoa era comparada com o valor real do IMC obtido pelas medidas antropométricas feitas anteriormente. Assim, com essa análise, é percebida a concepção de imagem corporal que cada pessoa tem de si mesma, podendo ter uma noção caso o indivíduo esteja passando por alterações alimentares (Fehrman-Rosas et al., 2016).

Dos 633 estudantes que foram analisados por Fehrman-Rosas et al. (2016), 21,4\% tinham aproximadamente o mesmo IMC comparando a imagem corporal com o modelo anatômico citado anteriormente, 68,7\% superestimaram seu peso e 9,7\% subestimaram seu peso. Dessa maneira, pode-se fazer a relação de que aqueles que superestimaram seu peso, além de terem uma imagem corporal alterada, associado com uma alimentação negativa, podem muitas vezes serem acompanhados de práticas maléficas à saúde, como a indução do vômito. Do mesmo modo, aqueles que subestimaram seu peso, podem ser afetados por fatores que os façam enxergar um peso menor do que elas realmente têm, o que pode levar a um consumo compulsivo de alimento.

Azzouzi et al. (2019) mostra também uma estreita correlação entra a imagética corporal e o comportamento alimentar. Com isso, a autocrítica está presente no quadro clínico de pacientes com critérios de diagnóstico para a anorexia nervosa. Como reforço, o estudo mostra que de $25 \%$ a $40 \%$ dos estudantes têm preocupação com a imagem corporal e tomam medidas para o controle do peso e da ingesta alimentar. Diante disso, apesar de não ter mostrado diferença significativa entre os sexos, é relatado que as mulheres demonstram maior preocupação quando se refere à insatisfação corporal.

Um estudo transversal conduzido com 227 estudantes universitários de ciências médicas, com a idade variando entre 
18 a 32 anos, mostrou que quase 9,7\% dos participantes eram casados e 90,3\% eram solteiros. Essa análise mostra que alguns fatores, como o relacionamento interpessoal, também podem influenciar na alteração da alimentação, por exemplo, em casos que a preocupação em iniciar um namoro leva o indivíduo a ter maior compromisso com a aparência física. Dessa forma, as pessoas tendem a iniciar métodos compulsivos de alimentação, seja com dietas exageradamente restritas ou qualquer tipo de iniciativa de redução do peso, mesmo que seja maléfica à saúde. Além disso, como a maioria dos estudantes são solteiros, essa preocupação e compulsão tende a ser ainda mais evidente (Hosseini et al., 2016).

A ortorexia nervosa, embora ainda não seja considerada uma doença, é caracterizada por fixação patológica com consumo saudável de alimentos, pensamentos perturbadores e preocupação excessiva e é avaliada através do ORTO-15, que é uma escala com 15 perguntas de 4 respostas graduadas (sempre, frequentemente, às vezes e nunca). Essa escala avalia o comportamento do indivíduo quanto à escolha, preparação, consumo, emoções e atitudes gerais relacionadas ao alimento dito saudável. Nesse sentido, um comportamento alimentar normal tem 4 como pontuação, e uma tendência ortoréxica é pontuada como 1. Dentre os 627 estudantes de medicina do Líbano pesquisados, 467 apresentaram pontuação para classificação como ortoréxicos, enquanto 160 apresentaram hábitos alimentares normais. Isso reforça, mais uma vez, o quanto o conhecimento na área da saúde pode ter tanto influências benéficas quanto maléficas nos aspectos alimentares (Farchakh et al., 2019). Esse mesmo estudo mostrou ainda que, a saúde mental piora após os alunos ingressarem no curso de medicina. Com isso, a ansiedade, que é um distúrbio comum nesses acadêmicos, tem influência direta nos aspectos alimentares, o que pode levar a tendências comportamentais de ortorexia, que mesmo que sejam sutis, podem evoluir para casos de scores cada vez mais baixos na escala ORTO-15 (Farchakh et al., 2019).

Em uma pesquisa feita por Hernández et al. (2019) com 628 estudantes da área médica, em que 193 eram do primeiro ano, 136 do segundo, 128 do terceiro ano, 81 do quarto ano e 90 do quinto ano, um total de 378 alunos tinham deficiência na qualidade do sono. Além disso, a frequência de sobrepeso e obesidade foi de apenas $41,87 \%$, sendo mais frequente em homens. Assim, pode ser feita uma relação entre a má qualidade do sono e o sobrepeso através da estatística, já que uma parcela desses alunos apresentava todos os dois distúrbios, o do sono e o de sobrepeso. Assim, esse estudo mostra que quando o tempo de duração do sono tende a ser menor do que seis horas, o sobrepeso tende a ser mais afetado entre os estudantes de medicina. Esse pensamento pode ser explicado pelo fato de que a redução do sono leva a uma menor disposição à prática de exercícios físicos, além de aumentar a ingestão calórica, principalmente durante à noite, gerando, assim, um círculo vicioso. Além disso, ligado ao fato de que os estudantes de medicina têm uma alta exposição à ansiedade, e muitas vezes, aulas práticas envolvendo plantões noturnos, tende a ser um grupo cada vez mais exposto à problemática em questão (Hernández et al., 2019).

Segundo outro estudo, a má qualidade no sono estaria envolvida na aprendizagem superficial dos estudantes universitários da área da saúde. Esse cronotipo irregular durante a noite estaria associado à ausência da realização do café da manhã, sugerindo uma possível relação com o aumento do risco para a obesidade (Mirghani et al., 2019). Outro estudo afirma que o sentimento de tristeza e a falta de interesse nas atividades comuns do dia a dia são os principais preditores dos distúrbios do sono, tais como a apneia do sono, transtornos afetivos, fadiga, insônia e pesadelos, uma vez que esses indivíduos relatam estar cansados ao acordarem de manhã e sentem sono durante a maior parte do dia (Abdulah \& Piro, 2018).

Além desses achados, o estudo de Abdulah \& Piro (2018) sugere que o consumo de bebidas alcoólicas pode estar relacionado ao desenvolvimento de distúrbios emocionais e síndrome das pernas inquietas durante a noite. Também, o tabagismo durante o período noturno contribuiu para que os pacientes tivessem movimentos corporais durante o sono. Outro fator contribuinte para o desenvolvimento de distúrbios mentais e aumento dos movimentos noturnos, seria o excesso de luz e barulho no quarto durante a noite, sendo identificados também, na população pesquisada, casos de sonambulismo. Esse cenário também foi verificado no estudo de Jniene et al. (2019), no qual evidenciou-se que os estudantes de medicina utilizavam 
dispositivos emissores de luz na hora de dormir, tinham má qualidade do sono e hábitos de vida não saudáveis, tais como má alimentação e sedentarismo, independente do sexo.

Já a pesquisa feita por Aldahash et al. (2018), afirmou que o aumento do IMC está relacionado ao consumo de fast food e irregularidade nos exercícios físicos. Outra correlação negativa importante feita por esse estudo foi entre o IMC e a duração do sono, mostrando que os estudantes fumantes eram mais propensos a serem obesos quando comparados ao que não fumavam e, consequentemente, tinham uma menor qualidade de sono.

No que se refere à prevalência, os distúrbios de sono, segundo Belingheri et al. (2020), são maiores entre estudantes da área da saúde do que na população em geral e entre estudantes de outras áreas. Nessa análise, em especial, foi encontrada uma maior prevalência de distúrbios do sono entre estudantes de enfermagem e medicina, pois estão, frequentemente, estressados devido à carga horária e à pressão da profissão.

Em outra perspectiva, a pesquisa de Althakafi et al. (2019) sugere que a diminuição na duração do sono poderia resultar em maior resistência à insulina, concomitante à queda do hormônio que suprime a ingesta alimentar denominado leptina e aumento do hormônio estimulante da fome, a grelina, o que ocorre por intermédio da regulação do eixo hipotálamoadrenal. Essa associação estaria envolvida com o surgimento de anormalidades metabólicas e aumento do peso. Esse mesmo estudo revelou que o sono irregular contribui para a elevação dos níveis de proteína C-reativa, a qual é um importante agente inflamatório marcador de doenças cardiovasculares.

Ainda de acordo com Althakafi et al. (2019), há uma estreita ligação entre a breve duração de sono e maiores chances de desencadear hiperlipidemias, acidentes automobilísticos e doenças arteriais coronarianas. Dados desse mesmo estudo afirmam a existência de uma associação entre o aumento da idade e a curta duração do sono, visto que, dentre os pesquisados, os participantes com idade superior a 30 anos dormiam menos do que 7 horas diárias de sono. Além do que, não houve diferença na duração do sono entre homens e mulheres. Verificou-se, também, que os participantes casados têm curta duração do sono quando comparados aos solteiros, isso se deve, provavelmente, ao aumento dos deveres e responsabilidades, incluindo o potencial de serem pais.

Outro estudo apresentou a relação da dor com o sono, sugerindo que deficiências no sono aumentam o risco de piorar o prognóstico para possíveis dores, uma vez que a percepção da dor é mais intensa durante a noite, o que acarreta um acúmulo de um efeito perturbador do sono. Isso resulta em ineficiência do sono e inquietação ao se deitar (Schlarb et al., 2017).

Além das consequências já expostas, outra evidência recente afirma que a cefaleia está intimamente relacionada a longos períodos sob forte pressão social e emocional, além do que, pacientes com dores de cabeça, são, comumente, mais depressivos e ansiosos que indivíduos que não sofrem com essa queixa. A cefaleia está relacionada ao desempenho acadêmico prejudicado, já que incapacita e aumenta a dificuldade dos estudantes de se concentrarem, limitando o sucesso acadêmico e influenciando, de forma negativa, o futuro profissional desses indivíduos. Prova disso seria a ausência frequente de estudantes com cefaleia às aulas em detrimento dos demais estudantes (Carneiro et al., 2019).

$\mathrm{Na}$ análise de Carneiro et al. (2019), citado anteriormente, os pesquisados relataram pelo menos um sintoma associado à cefaleia, dentre eles estão a sensibilidade à luz e ao som, náuseas ou vômitos. Outros sintomas foram referidos, porém em menor quantidade, tais como a descoordenação motora, afasia, dormência, perda de visão periférica, lipotimia, formigamento, irritabilidade, insônia e vertigem. Em relação ao consumo de fast foods, não houve relação desse consumo com o surgimento de cefaleias. Nesse mesmo estudo, evidenciou-se que a maioria dos estudantes com cefaleia eram mulheres e, dentre o total de indivíduos com cefaleia, a maioria relatou presença de histórico familiar dessa queixa e maior intensificação das crises após a entrada na universidade.

No que se refere à prevalência da cefaleia, outra pesquisa demonstrou que mais da metade da população universitária pesquisada era do gênero feminino. Além disso, do total de pesquisados, mais de $90 \%$ já se queixaram de cefaleia no mínimo 
uma vez na vida após o ingresso na universidade e dentre eles, a maioria alegou nunca ter se tratado com neurologista, afirmando ter realizado a automedicação com analgésicos comuns, os quais podem gerar reações de hipersensibilidade, sangramento digestivo e mascarar doenças passíveis de progressão. Ainda, nesse mesmo trabalho, cerca de $80 \%$ dos pesquisados relacionaram o surgimento da cefaleia com o estresse (Santos et al., 2019).

O estudo de Landel \& Dasgupta (2018) afirmou que a Síndrome de Burnout tem maior prevalência na população médica do que nas outras profissões, o que poderia ser facilmente evitada se houvesse uma maior flexibilidade do cronograma. O recomendado, segundo os autores, seria a implementação de uma rotina regular de sono, evitar o uso de estimulantes, evitar medicamentos que auxiliam o sono, introduzir uma dieta saudável, incluindo ingesta proteica e carboidratos complexos, e praticar exercícios. Outras opções para uma possível melhora da qualidade do sono seria o desligamento de dispositivos eletrônicos, dormir em um quarto escuro, fresco e silencioso e usar tampões de ouvido, se necessário. Já ao acordar, seria recomendado usar uma fonte de luz brilhante para estimular o ciclo da vigília. Esse panorama de recomendações para uma melhora do estilo de vida dos universitários da área da saúde também foi mencionado no estudo de Mirghani et al. (2015).

Uma análise feita por Qiu et al. (2019) mostrou que, quanto à saúde dos estudantes de medicina, os alunos do internato, isto é, nos dois últimos anos do curso, apresentaram menor qualidade de vida em relação aos estudantes do mesmo curso, mas em anos distintos. Isso pode ter acontecido devido ao provável aumento da carga horária de estudos, com aumento do número de curso de especialização e estágios, em detrimento dos estudos dos ciclos básico e clínico, que estudam a teoria em maior proporção quando comparada à prática. Nesse mesmo estudo, ficou evidente que o desenvolvimento de forças psicológicas positivas, como esperança, resiliência e otimismo em estudantes de medicina deve ser realizado a fim de amenizar os sofrimentos advindos da profissão. Além disso, observou-se que a adoção de novas formas de aprendizagem no curso, como as salas de aulas invertidas, demonstra melhorar a motivação dos estudantes e o desempenho nos exames. Também, nesse mesmo estudo, foi verificado que os relacionamentos com colegas de quarto estão associados a uma melhoria da qualidade de vida desses estudantes. Para que esse relacionamento seja positivo, é necessário que haja empatia e compreensão entre esses indivíduos, além de ser essencial a organização e a limpeza adequada dos dormitórios. As atividades em grupo e a satisfação de fazer vínculos com os amigos e familiares podem sugerir uma melhora significativa na qualidade de vida dos estudantes universitários e, consequentemente, uma melhora no desenvolvimento na vida acadêmica.

\section{Considerações Finais}

Os resultados apresentados nesse estudo evidenciam as diferenças entre o estilo de vida e o recomendado, sobretudo em relação ao sono e a ingesta de alimentos, relacionando, ainda, queixas comuns entre os estudantes, como a cefaleia. Foram apresentados os diversos fatores de risco para doenças crônicas não transmissíveis em universitários da área da saúde, dentre as quais destacam-se neoplasias, distúrbios metabólicos, doenças dos sistemas nervoso, respiratório, cardiovascular e osteoarticular, principalmente. No que tange aos fatores de risco, foram destacados a insuficiência do sono, a má alimentação, o sedentarismo, o consumo de álcool e o tabagismo.

No entanto, apesar de todos os efeitos negativos da má qualidade do sono e de uma dieta irregular, esses fatores de risco podem ser gerenciados com a ajuda da medicina preventiva no nível da atenção primária, por meio de educação em saúde aos universitários em questão. É importante que esses aconselhamentos sejam acompanhados da desestimulação da automedicação e de mecanismos prejudiciais referentes à alimentação e ao sono, para que possíveis danos à saúde possam ser evitados. Além disso, seria essencial o engajamento conjunto do governo, o qual poderia desviar mais esforços para a educação física entre os universitários, o que colaboraria na diminuição do sedentarismo nessa população.

Para que haja maior esclarecimento entre os fatores de risco e potenciais consequências, são necessários mais estudos acerca dessa temática, sobretudo com amostras maiores, para que possa confirmar com mais certeza o impacto dessas 
patologias secundárias ao estilo de vida inadequado. Contudo, os dados já existentes podem ser usados como base para orientar futuras pesquisas científicas que investigam a associação do ciclo sono-vigília alterado pela má qualidade do sono e a dieta inadequada entre universitários.

\section{Referências}

Abdulah, D. M., \& Piro, R. S. (2018). Sleep disorders as primary and secondary factors in relation with daily functioning in medical students. Annals of Saudi medicine, 38(1), 57-64. https://doi.org/10.5144/0256-4947.2018.57

Ahmed, J., Alnasir, F., Jaradat, A., Al Marabheh, A. J., \& Hamadeh, R. R. (2019). Association of Overweight and Obesity with High Fast Food Consumption by Gulf Cooperation Council Medical Students. Ecology of food and nutrition, 58(5), 495-510.

Aldahash, F. D., Alasmari, S. A., Alnomsi, S. J., Alshehri, A. M., Alharthi, N. F., Aloufi, A., Al Atawi, M. S., Alotaibi, A. A., \& Mirghani, H. O. (2018). Relationship of body mass index to sleep duration, and current smoking among medical students in Tabuk City, Saudi Arabia. Electronic physician, 10(9), 7273-7278. https://doi.org/10.19082/7273

Althakafi, K. A., Alrashed, A. A., Aljammaz, K. I., Abdulwahab, I. J., Hamza, R., Hamad, A. F., \& Alhejaili, K. S. (2019). Prevalence of short sleep duration and effect of co-morbid medical conditions - A cross-sectional study in Saudi Arabia. Journal of family medicine and primary care, 8(10), 3334-3339. https://doi.org/10.4103/jfmpc.jfmpc_660_19

Azzouzi, N., Ahid, S., Bragazzi, N. L., Berhili, N., Aarab, C., Aalouane, R., Boujraf, S., \& Rammouz, I. (2019). Eating disorders among Moroccan medical students: cognition and behavior. Psychology research and behavior management, 12, 129-135. https://doi.org/10.2147/PRBM.S165114

Belingheri, M., Pellegrini, A., Facchetti, R., De Vito, G., Cesana, G., \& Riva, M. A. (2020). Self-reported prevalence of sleep disorders among medical and nursing students. Occupational medicine (Oxford, England), 70(2), 127-130. https://doi.org/10.1093/occmed/kqaa011

Bezerra, M. P. P., Lira, R. C. M., Silva, A. O., Melo, I. L. P., Silva, N. S., Roque, T. S. (2020). A influência da variação no ciclo do sono sobre o desempenho universitário e o bem-estar de estudantes de Medicina. Research, Society and Development, 9(12), e28091211057. http://dx.doi.org/10.33448/rsd-v9i12.11057

Carneiro, A. F., Cavalcante Neto, P. G., Ferreira, J. F. I. S., Garcia, B. F., Silva, F. de A. C., \& Leal, P. R. L. (2019). A prevalência de cefaleia e fatores psicossociais associados em estudantes de medicina no Ceará. Revista De Medicina, 98(3), 168-179. https://doi.org/10.11606/issn.1679-9836.v98i3p168-179

Choque Zurita, R., Daza Cazana, L., Philco Lima, P., Gonzáles, L., \& Alanes Fernández, Á. (2018). Factores de riesgo para enfermedades crónicas no transmisibles en estudiantes de medicina de la universidad mayor de san andrés (umsa), La Paz - Bolivia 2015. Rev. méd., (La Paz); 24(1): 5-12.

El-Kassas, G., \& Ziade, F. (2016). Exploration of the Dietary and Lifestyle Behaviors and Weight Status and Their Self-Perceptions among Health Sciences University Students in North Lebanon. BioMed research international, 9762396. https://doi.org/10.1155/2016/9762396

Farchakh, Y., Hallit, S., \& Soufia, M. (2019). Association between orthorexia nervosa, eating attitudes and anxiety among medical students in Lebanese universities: results of a cross-sectional study. Eating and weight disorders: EWD, 24(4), 683-691. https://doi.org/10.1007/s40519-019-00724-6

Fehrman-Rosas, Pamela, Delgado-Sánchez, Claudia, Fuentes-Fuentes, Jessica, Hidalgo-Fernández, Andrea, Quintana-Muñoz, Carol, Yunge-Hidalgo, Wilma, Fernández-Godoy, Eloina, \& Durán-Agüero, Samuel. (2016). Asociación entre autopercepción de imagen corporal y patrones alimentarios en estudiantes de Nutrición y Dietética. Nutrición Hospitalaria, 33(3), 649-654. https://dx.doi.org/10.20960/nh.274

Ferreira, M. A. A., Domingos, S. R. A., Trovão, C. B. A., Domiciano, D. C., Silva, B. A. da, Rocha, L. G., Carmello, L. do M., \& Soares, E. A. (2020). Perfil da Qualidade de Sono de um grupo de estudantes de Medicina. Research, Society and Development, 9(9), e718997806, DOI: 10.33448/rsd-v9i9.7806.

Gonçalves, L. F., \& Haas, P. (2020). Impacto da alimentação associada ao hábito do sono: uma revisão sistemática. Research, Society and Development, 9(11), e57791110238. DOI: $10.33448 /$ rsd-v9i11.10238.

Hernández, A. O., Turrubiartes, G. P., Castillo, A. P., Aldrett, F. P., Sandoval, J. I. R., González, J. F. A., Rosas, C. A. H., Castañeda, F. A. M., Pulido, I. K. R., Zamarrón, A. D. S. \& Hernández-Sierra, J. F. (2019). Asociación entre la deficiencia de sueño y sobrepeso y obesidad en estudiantes de medicina de nueva generación de México: un cambio de paradigma. Rev Esp Nutr Comunitaria, 25(4).

Hosseini, S. N., Emdadi, S., Jalilian, F., Karami Matin, B., Ataee, M., \& Mirzaei Alavijeh, M. (2016). Fitness Intention and Its Relationship With Eating Attitudes: A Cross-Sectional Study of Iranian Female Medical College Students. Iranian journal of psychiatry and behavioral sciences, 10(1), e4307. https://doi.org/10.17795/ijpbs-4307

Jaremków, A., Markiewicz-Górka, I., \& Pawlas, K. (2020). Assessment of health condition as related to lifestyle among students in the examination period. International Journal of Occupational Medicine and Environmental Health, 33(3), 339-351. https://doi.org/10.13075/ijomeh.1896.01563

Jniene, A., Errguig, L., El Hangouche, A. J., Rkain, H., Aboudrar, S., El Ftouh, M., \& Dakka, T. (2019). Perception of Sleep Disturbances due to Bedtime Use of Blue Light-Emitting Devices and Its Impact on Habits and Sleep Quality among Young Medical Students. BioMed research international, 2019, 7012350. https://doi.org/10.1155/2019/7012350

Kanikowska, D., Sikorska, D., Kuczyńska, B., Grzymisławski, M., Bręborowicz, A., \& Witowski, J. (2017). Do medical students adhere to advice regarding a healthy lifestyle? A pilot study of BMI and some aspects of lifestyle in medical students in Poland. Advances in clinical and experimental medicine: official organ Wroclaw Medical University, 26(9), 1391-1398. https://doi.org/10.17219/acem/65783

Kowalska, M., Żbikowska, A., Onacik-Gür, S., \& Kowalska, D. (2017). Acrylamide in food products - eating habits and consumer awareness among Medical School students. Ann Agric Environ Med., 24(4), 570-574. https://doi.org/10.5604/12321966.1232764 
Research, Society and Development, v. 10, n. 2, e23710212393, 2021

(CC BY 4.0) | ISSN 2525-3409 | DOI: http://dx.doi.org/10.33448/rsd-v10i2.12393

Landel, M., \& Dasgupta, S. (2018). Sleep hygiene practices and its effects on job satisfaction in emergency medicine physicians and physicians-in-training. The American journal of emergency medicine, 36(11), 2118-2119. https://doi.org/10.1016/j.ajem.2018.03.045

Mirghani, H. O., Ahmed, M. A., \& Elbadawi, A. S. (2015). Daytime sleepiness and chronic Sleep deprivation effection on academic performance among Sudanese medical students. Journal of Taibah University Medical Sciences, 1-4. https://doi.org/10.1016/j.jtumed.2015.05.003

Mirghani, H. O., Albalawi, K. S., Alali, O. Y., Albalawi, W. M., Albalawi, K. M., Aljohani, T. R., \& Albalawi, W. S. (2019). Breakfast skipping, late dinner intake and chronotype (eveningness-morningness) among medical students in Tabuk City, Saudi Arabia. The Pan African medical journal, $34,178$. https://doi.org/10.11604/pamj.2019.34.178.16250

Pereira A. S., et al. (2018). Metodologia da pesquisa científica. [e-book]. Santa Maria. Ed. UAB/NTE/UFSM. Disponível em: https://repositorio.ufsm.br/bitstream/handle/1/15824/Lic_Computacao_Metodologia-Pesquisa-Cientifica.pdf?sequence=1.

Qiu, Y., Yao, M., Guo, Y., Zhang, X., Zhang, S., Zhang, Y., Huang, Y., \& Zhang, L. (2019). Health-Related Quality of Life of Medical Students in a Chinese University: A Cross-Sectional Study. International journal of environmental research and public health, 16(24), 5165. https://doi.org/10.3390/ijerph16245165

Santos, R., Rêgo, R. C. S., Santos, V. L. B., \& Prado, M. R. (2019). Prevalência de cefaleia e seus impactos em estudantes de medicina em uma universidade pública. Revista Brasileira de Neurologia, 55(3), 5-8.

Schlarb, A. A., Claßen, M., Hellmann, S. M., Vögele, C., \& Gulewitsch, M. D. (2017). Sleep and somatic complaints in university students. Journal of Pain Research, 10, 1189-1199.

Tamayo, C. C., Alcocer, R. H., Choque, S. L., Chuquimia, N. A., Condori, S. P., Gutierrez, F. I. et al. (2016). Factores y determinantes del consumo de comida chatarra en estudiantes de la facultad de medicina, enfermería, nutrición y tecnología medica, La Paz- Bolivia 2016. Cuad. - Hosp. Clín., 57(3): 31-40.

Torres-Mallma, C., Trujillo-Valencia, C., Urquiza-Díaz, A. L., Salazar-Rojas, R., \& Taype-Rondán, A. (2016). Hábitos alimentarios en estudiantes de medicina de primer y sexto año de una universidad privada de Lima, Perú. Revista chilena de nutrición, 43(2), 146-154. https://dx.doi.org/10.4067/S071775182016000200006 